\title{
Pharmacogenomics in oncology care
}

\author{
Kelly K. Filipski ${ }^{1}$, Leah E. Mechanic ${ }^{1}$, Rochelle Long ${ }^{2}$ and Andrew N. Freedman ${ }^{1}$ \\ 1 Epidemiology and Genomics Research Program, Division of Cancer Control and Population Sciences, National Cancer Institute, Rockville, MD, USA \\ 2 Pharmacological and Physiological Sciences Branch, Division of Pharmacology, Physiology, and Biological Chemistry, National Institute of General Medical \\ Sciences, Bethesda, MD, USA
}

\section{Edited by:}

Luis Abel Quiñones, University of

Chile, Chile

Reviewed by:

Elena García-Martín, Universidad de Extremadura, Spain

Angela Roco, Servicio de Salud Metropolitano Occidente, Chile

*Correspondence:

Kelly K. Filipski, Epidemiology and Genomics Research Program,

Division of Cancer Control and

Population Sciences, National

Cancer Institute, 9609 Medical

Center Dr. Room 4E228, Rockville,

MD 20850, USA

e-mail: kelly.filipski@nih.gov
Cancer pharmacogenomics have contributed a number of important discoveries to current cancer treatment, changing the paradigm of treatment decisions. Both somatic and germline mutations are utilized to better understand the underlying biology of cancer growth and treatment response. The level of evidence required to fully translate pharmacogenomic discoveries into the clinic has relied heavily on randomized control trials. In this review, the use of observational studies, as well as, the use of adaptive trials and next generation sequencing to develop the required level of evidence for clinical implementation are discussed.

Keywords: pharmacogenomics, pharmacogenetics, somatic, germline, targeted therapy

\section{INTRODUCTION}

In the last decade, the use of genomics in oncology significantly impacted treatment decisions for many patients. Oncologists have a variety of treatment options; however one patient may experience serious adverse events whereas another patient receives no therapeutic effect. Within a population, substantial variation exists resulting in unpredictable responses. Pharmacogenomics, the study of the interaction between the genome and clinical drug response (Monte et al., 2012), evaluates the associations between drug efficacy and toxicity and variation in drug metabolizing enzymes, receptors, transporters, and drug targets (Crews et al., 2012). The main priority of pharmacogenomics is to optimize treatment by understanding the underlying biological mechanisms and utilizing genomic contributions to treatment response to predict and individualize therapy and improve treatment outcomes. Unlike other diseases, cancer genetics must take into account both acquired (somatic) and inherited (germline) variation, both of which contribute to the efficacy and safety of a drug. However to date, integrated studies of germline and somatic variation have been limited. Somatic mutations are often attributed to treatment efficacy, whereas germline mutations are used to identify patients at highest risk of developing serious adverse events (Gillis et al., 2014). In this review, we will discuss examples of pharmacogenomic markers and differences in the evidence level required for implementation into clinical care. In addition, several mechanisms for developing evidence for clinical implementation and new technologies entering oncology practice will be discussed.

\section{SOMATIC MUTATIONS AND TARGETED THERAPY}

Somatic mutations have highlighted the importance of understanding the underlying biology of cancer with discoveries elucidating the primary genetic changes driving tumorigenesis providing molecular drug targets. Prospective tumor sequencing is being increasingly utilized, changing the paradigm of cancer treatment from site specific cytotoxic treatment, to molecularly targeted treatment (MacConaill et al., 2011; Ong et al., 2012). Many drugs are being developed for defined molecular targets (Simon, 2013), one such example is the use of crizotinib in anaplastic lymphoma kinase (ALK) positive non-small cell lung cancer (NSCLC). Tumor DNA sequencing identified two patients with NSCLC harboring novel ALK rearrangements as crizotinib, a tyrosine kinase inhibitor, moved into clinical trials. Both patients showed marked response to crizotinib prompting protocol amendments to prospectively test for $A L K$ rearrangements throughout clinical development (Ou, 2011; Ou et al., 2012). The fortuitous discovery of $A L K$ rearrangements during phase I trials of crizotinib restricted development to a subset of patients relying heavily on rigorous randomized controlled trials with an appropriate companion diagnostic to select patients. Predictive testing for biomarkers like $A L K$ reduces unnecessary treatment in patients that will not respond and helps avoid potentially toxic effects of treatment (Ong et al., 2012). Molecularly targeted therapies like crizotinib have replaced cytotoxic therapy as standard of care in several cancer types including breast cancer, NSCLC, and melanoma (Ong et al., 2012; Gillis et al., 2014). Randomized clinical trials (RCTs) have been important to modern medicine, however the shift away from average treatment effects within a whole population to molecularly defined sub-populations is new to clinical trial design, and will be discussed later in this review.

\section{GERMLINE MUTATIONS}

In oncology, germline mutations play a significant role in the treatment response to both chemotherapy and targeted anticancer agents. These mutations are often associated with the pharmacokinetics of a drug contributing to treatment related 
adverse events experienced by patients (Hertz and McLeod, 2013; Gillis et al., 2014). In this regard, germline pharmacogenomic markers can identify patients at highest risk of developing serious adverse events that could subsequently lead to treatment discontinuation and failure like musculoskeletal pain after treatment with aromatase inhibitors. Severe musculoskeletal pain has been reported in up to half of women treated with aromatase inhibitors contributing to a treatment discontinuation rate of about $10 \%$ (Crew et al., 2007; Henry et al., 2008; Ingle et al., 2010). Ingle et al. found four single nucleotide polymorphisms (SNPs) mapping to the T-cell leukemia 1A (TCL1A) gene were associated with the development of musculoskeletal adverse events in patients receiving adjuvant aromatase inhibitors (Ingle et al., 2010). Subsequent functional studies revealed that TCL1A was induced by estrogen with higher levels of expression in cells with the variant alleles for these SNPs. Further results suggested an estrogen dependent, TCL1A SNP-dependent regulation of cytokines, cytokine receptors, and NF- $\mathrm{BB}$ transcriptional activity. These SNP-dependent changes may help to elucidate the pathway involved in musculoskeletal pain following aromatase inhibitor mediated estrogen deprivation (Liu et al., 2012). The strategy of discovering genetic variants and studying the underlying biology of the association is central in pharmacogenomic studies. It outlines a strong biological basis for the genetic association and provides mechanistic insight into the biology of the event that could lead to new drug targets to prevent the toxicity. Pharmacogenomic markers like TCL1A are extremely important when taken into context with not only the large number of women that could be exposed to aromatase inhibitors, but the fact that many of those women will have long term survival after receiving aromatase inhibitors and may experience decreased quality of life due to musculoskeletal pain. However, like many pharmacogenomic markers, TCL1A may never be used in clinical practice because a large randomized clinical trial will never be completed to study the association, even though other treatment options are available and with the understanding of the biology prevention strategies could be developed.

In addition to adverse events and pharmacokinetics of a drug, germline mutations may influence drug efficacy. Recently a germline mutation in the proapoptotic gene BIM was associated with the resistance to tyrosine kinase inhibitors in chronic myeloid leukemia (CML) and epidermal growth factor receptor (EGFR) mutant NSCLC. Identification of this mutation not only explains some of the poor response seen in patients with CML treated with imatinib, but also provides biological insight into different strategies to overcome the resistance that are currently in preclinical testing (Cheng and Sawyers, 2012; Ng et al., 2012). Although still in development, BIM is an important reminder that only focusing on somatic or germline variation investigators can miss key mutations that affect treatment outcomes.

One of the most well known pharmacogenomic markers is the association of thiopurine-S-methyltransferase (TPMT) and mercaptopurine (6-MP). Mercaptopurine is an important component of pediatric acute lymphoblastic leukemia (ALL) treatment, and is used in the treatment of some nonmalignant diseases (Paugh et al., 2011). A variant in the TPMT gene reduces the function of the enzyme leading to excessive levels of cytotoxic thioguanine nucleotides (6-TGNs) subsequently leading to an increased risk of severe myelosuppression (Paugh et al., 2011). Although a randomized clinical trial has never been done, the Food and Drug Administration (FDA) agreed that the evidence was sufficient to mention testing for TPMT deficiency, thus allowing identification of safe doses of mercaptopurine without compromising efficacy (Relling et al., 2011). Ample evidence, including in vitro and retrospective analyses provide support for the use of TPMT testing in patients receiving mercaptopurine to prevent serious treatment induced myelosuppression (Relling et al., 2011), but the consistent and widespread use of pre-treatment TPMT testing has not been universally accepted.

\section{STRATEGIES FOR DEVELOPING PHARMACOGENOMIC EVIDENCE}

The field of pharmacogenomics has uncovered an abundance of actionable and clinically relevant markers including both somatic (Table 1) and germline (Table 2) mutations. Prospective screening for predictive pharmacogenomic markers, like TPMT, may enhance treatment response by reducing the risk of toxicity from systemic drug concentrations while maintaining the anti-cancer activity of the drug. However, implementation into clinical practice has been slow, in part due to contradictory professional guidelines and recommendations, and differing thresholds for evidence (Gillis et al., 2014). RCTs have been the gold standard for evidence in treatment response and genetic testing and for pharmacogenomic markers like ALK that were discovered during the drug development process RCTs are feasible. Randomization in experimental studies attempts to control for biases by balancing factors that affect outcomes across study groups. However, clinical trials are not always the most feasible approach for developing evidence due to cost, time constraints, and the large sample size necessary to complete a trial (Gillis et al., 2014). Observational studies are more prone to a number of biases and the potential confounding which may lead to incorrect results. But these studies offer some advantages over clinical trials including larger numbers of subjects at an affordable cost, ability to examine meaningful genomic subgroups, longer follow-up times, and ability to examine drugs and their interactions with the genome that are used off-label (Dreyer et al., 2010). Rigorously designed high-quality observational studies can play a particularly important role in developing evidence for decision making in cancer pharmacogenomics because they can relatively quickly generate results that are applicable to real-world situations and examine long-term risk and benefits. Below we discuss the use of epidemiologic studies for developing evidence to support the use of pharmacogenomic tests where RCTs are not an option.

\section{RETROSPECTIVE OBSERVATIONAL STUDY}

Leveraging clinical trial data in a retrospective case-control study, where all the patients are receiving treatment, the cases and controls are those affected and unaffected by the outcome of interest, is a powerful design approach for detecting common variants associated with phenotypic traits (Ritchie, 2012). These retrospective analyses have proven successful for both somatic and germline mutations. Cetuximab, an anti-EGFR monoclonal 
Table 1 | Selected somatic pharmacogenomic markers.

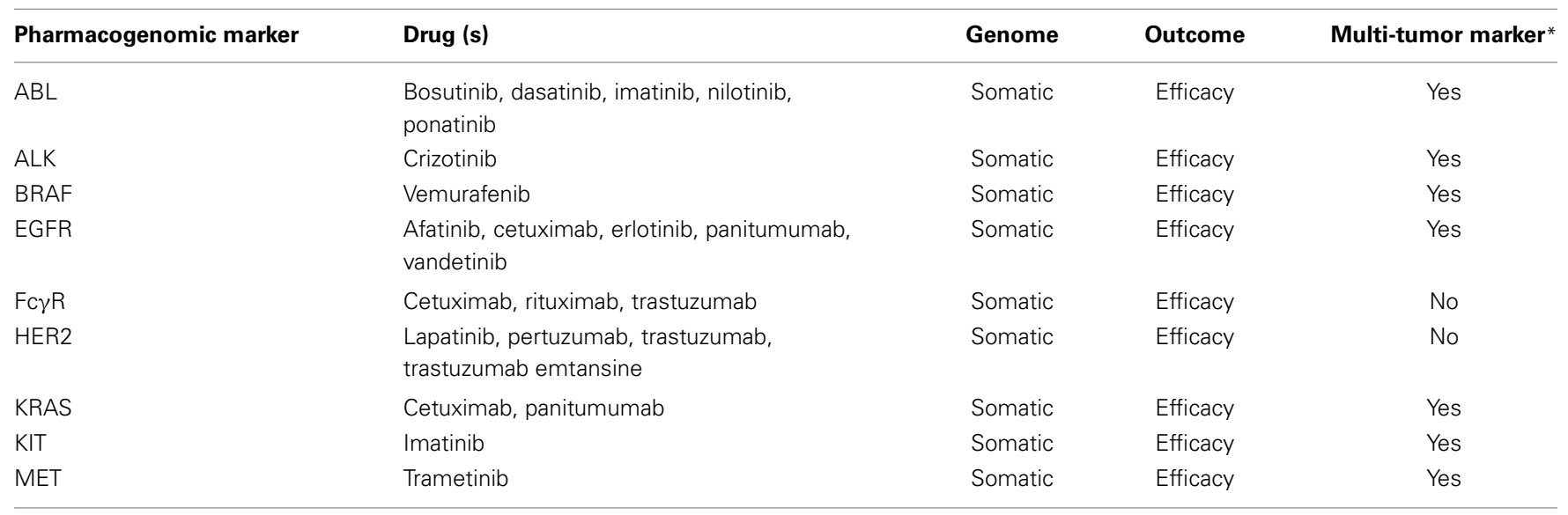

* Commercially available multi-marker tumor panels.

Arup Laboratories (http://Itd.aruplab.com/Tests/Pub/2007991).

AsuraGen (http://asuragen.com/products-and-services/genomic-services/next-generation-sequencing-services/).

Foundation Medicine (http://www.foundationone.com/).

Table 2 | Selected germline pharmacogenomic markers.

\begin{tabular}{|c|c|c|c|c|}
\hline Pharmacogenomic marker & Drug (s) & Genome & Outcome & Multi-tumor marker* \\
\hline BIM & Imatinib & Germline & Efficacy & No \\
\hline CYP2B6 & Cyclophosphamide & Germline & Toxicity & No \\
\hline DPYD & Capecitabine, fluorouracil & Germline & Toxicity & No \\
\hline G6PD & Rasburicase & Germline & Toxicity & No \\
\hline SLC28A3 & Anthracyclines & Germline & Toxicity & No \\
\hline TCL1A & Aromatase inhibitors & Germline & Toxicity & No \\
\hline TPMT & Mercaptopurine, thioguanine, cisplatin & Germline & Toxicity & No \\
\hline UGT1A1 & Irinotecan & Germline & Toxicity & No \\
\hline
\end{tabular}

${ }^{*}$ Commercially available multi-marker tumor panels.

Arup Laboratories (http://ltd.aruplab.com/Tests/Pub/2007991).

AsuraGen (http://asuragen.com/products-and-services/genomic-services/next-generation-sequencing-services/).

Foundation Medicine (http://www.foundationone.com/).

antibody is used in the treatment of colorectal cancer, however only a subset of patients responded to treatment. Tumor samples from cetuximab treated patients were retrospectively analyzed identifying mutations in the KRAS gene that were associated with response to therapy (Karapetis et al., 2008; Van Cutsem et al., 2009). Patients that are mutation positive do not respond to cetuximab, leading to the development of a companion diagnostic for KRAS testing and changes to the prescribing information for cetuximab to only be used in KRAS mutation negative patients. Although only retrospective analyses were performed, professional societies and the FDA recommend KRAS testing prior to initiation of cetuximab therapy (Kelley et al., 2011; Evaluation of Genomic Applications in Practice and Prevention Working Group, 2013).

\section{ELECTRONIC MEDICAL RECORDS}

The electronic medical record (EMR) provides a wealth of information to study questions at the population level. Large patient cohorts can be quickly created for a variety of diseases and drug response phenotypes to better evaluate drug safety. With the establishment of biobanks concurrent with the adoption of EMR, studies of genomic associations of drug response may become more comprehensive. The NHGRI funded Electronic Medical Records and Genomics (eMERGE) Network is leading the way by developing best practices for EMR guided genomic studies (Gottesman et al., 2013). These population based studies have access to much larger populations of patients making it easier to study rare, but serious, adverse events. One eMERGE site, Marshfield Clinic Personalized Medicine Project (PMRP), 
identified genetic factors associated with thromboembolic events in women taking tamoxifen. Patients were identified for a study by interrogating the EMR for breast cancer diagnosis, tamoxifen treatment, and thromboembolic event. SNPs in the ESR1 gene were shown to be associated with thromboembolic events in women treated with tamoxifen. These events are relatively rare $(1.7-8.4 \%)$ therefore using this large practice based cohort, investigators were able to capture enough events to evaluate the underlying genetic difference in women with and without thromboembolic events (Onitilo et al., 2009).

EMR based studies provide some advantages over RCTs in evaluating drug safety in the general population. Biobanks of several sites can participate together in investigations, thus increasing sample size and study power. In addition, clinical trials exclude patients with comorbidities, taking concomitant medications, and may include run in periods excluding patients that show drug intolerance prior to randomization. In the unrestricted EMR populations a higher incidence of adverse events may be identified (Wilke et al., 2011).

\section{EMERGING TECHNOLOGIES AND TRIAL DESIGN}

In addition to retrospective analyses and EMR studies to develop evidence for the use of pharmacogenomic tests in clinical practice, the acceptance of new technologies and clinical trial designs are imperative to improving clinical outcomes. Both the time and cost of genotyping has been steadily decreasing allowing for the creation of denser genotyping panels and advent of next generation sequencing (NGS) has allowed for a more complete view of the genome. There are three types of NGS: whole exome sequencing, whole genome sequencing, and targeted gene panels (Rehm et al., 2013). Unlike genotyping of candidate genes and genome wide association studies, WGS can identify rare variants that may be very important in the genomic contribution to treatment response and toxicity (Gillis et al., 2014), particularly for a drug like methotrexate that has wide inter-individual differences. A GWAS identified SNPs annotated to the transporter gene, SLCO1B1 that were associated with methotrexate clearance (Trevino et al., 2009). Deep resequencing of the gene was done and Ramsey et al. found that rare variants, only identified through deep sequencing accounted for $17.8 \%$ of the genes effect on clearance (Ramsey et al., 2012). NGS can provide not only common variants, but a comprehensive catalog of rare variants that may have a larger effect size than common variants contributing to the overall phenotype of drug response (Gillis et al., 2014). There are some challenges with NGS, particularly the daunting task of handling and interpreting NGS results.

With the advent of molecularly targeted therapies, prospective use of NGS is being incorporated into adaptive clinical trials, using the genomic landscape of the tumor to direct therapy as opposed to site specific treatment (MacConaill et al., 2011; Ong et al., 2012). Trials such as the National Cancer Institute's MPACT trial are prospectively evaluating tumor mutations for actionable variants and then randomizing patients to receive a drug targeting the aberrant pathway or standard of care regardless of tumor site (Simon and Polley, 2013). Forward thinking trials like MPACT are poised to change the way tumors are classified and patients are treated. Already targeted gene panels have emerged focused on a limited set of genes creating a greater depth of coverage and improving the ability to interpret the findings in the clinic (Rehm et al., 2013). Commercially available multi-tumor marker panels are primarily focused on somatic mutations with the inclusion of several known driver mutations predictive of treatment response to molecularly targeted therapies (Table 1), however to date, it is unknown how comprehensive these panels are, whether they are including the optimal set of genes, and the broader utility of these panels has yet to be evaluated. There are challenges and limitations to the use of NGS, but NGS and multi-marker tumor panels may provide an opportunity to generate incredible amounts of information about the tumor genome and thus drive research to elucidate some of the biological mechanisms underlying the tumor growth and survival.

\section{CONCLUSION}

Pharmacogenomics has provided significant impact in oncology treatment, but implementation differs between somatic and germline markers. Developing sufficient evidence for use of pharmacogenomic markers has been difficult, in part because RCTs cannot be performed for every marker identified. A move toward retrospective analyses, large population studies using EMRs, and the establishment of mechanism-based evidence can propel progress in the field. In addition, embracing new methods, such as NGS and adaptive clinical trials, is providing a wealth of information about tumor biology and changing the landscape of cancer treatment. To date, pharmacogenomic studies tend to focus on either somatic or germline mutations in isolation from each other, but to truly optimize clinical care both genomes need to be integrated into clinical decision making. Both well designed, forward thinking trials and population based studies are necessary to fully implement pharmacogenomics into clinical care.

\section{REFERENCES}

Cheng, E. H., and Sawyers, C. L. (2012). In cancer drug resistance, germline matters too. Nat. Med. 18, 494-496. doi: 10.1038/nm.2725

Crew, K. D., Greenlee, H., Capodice, J., Raptis, G., Brafman, L., Fuentes, D., et al. (2007). Prevalence of joint symptoms in postmenopausal women taking aromatase inhibitors for early-stage breast cancer. J. Clin. Oncol. 25, 3877-3883. doi: 10.1200/JCO.2007.10.7573

Crews, K. R., Hicks, J. K., Pui, C. H., Relling, M. V., and Evans, W. E. (2012). Pharmacogenomics and individualized medicine: translating science into practice. Clin. Pharmacol. Ther. 92, 467-475. doi: 10.1038/clpt.2012.120

Dreyer, N. A., Tunis, S. R., Berger, M., Ollendorf, D., Mattox, P., and Gliklich, R. (2010). Why observational studies should be among the tools used in comparative effectiveness research. Health Aff. (Millwood) 29, 1818-1825. doi: 10.1377/hlthaff.2010.0666

Evaluation of Genomic Applications in Practice and Prevention Working Group. (2013). Recommendations from the EGAPP Working Group: can testing of tumor tissue for mutations in EGFR pathway downstream effector genes in patients with metastatic colorectal cancer improve health outcomes by guiding decisions regarding anti-EGFR therapy? Genet. Med. 15, 517-27. doi: 10.1038/gim.2012.184

Gillis, N., Patel, J., and Innocenti, F. (2014). Clinical implementation of germ line cancer pharmacogenetic variants during the next-generation sequencing era. Clin. Pharmacol. Ther. 95, 269-280. doi: 10.1038/clpt.2013.214

Gottesman, O., Kuivaniemi, H., Tromp, G., Faucett, W. A., Li, R., Manolio, T. A., et al. (2013). The Electronic Medical Records and Genomics (eMERGE) Network: past, present, and future. Genet. Med. 15, 761-771. doi: 10.1038/gim.2013.72

Henry, N. L., Giles, J. T., Ang, D., Mohan, M., Dadabhoy, D., Robarge, J., et al. (2008). Prospective characterization of musculoskeletal symptoms in early stage 
breast cancer patients treated with aromatase inhibitors. Breast Cancer Res. Treat. 111, 365-372. doi: 10.1007/s10549-007-9774-6

Hertz, D. L., and McLeod, H. L. (2013). Use of pharmacogenetics for predicting cancer prognosis and treatment exposure, response and toxicity. J. Hum. Genet. 58, 346-352. doi: 10.1038/jhg.2013.42

Ingle, J. N., Schaid, D. J., Goss, P. E., Liu, M., Mushiroda, T., Chapman, J. A., et al. (2010). Genome-wide associations and functional genomic studies of musculoskeletal adverse events in women receiving aromatase inhibitors. J. Clin. Oncol. 28, 4674-4682. doi: 10.1200/JCO.2010.28.5064

Karapetis, C. S., Khambata-Ford, S., Jonker, D. J., O'callaghan, C. J., Tu, D., Tebbutt, N. C., et al. (2008). K-ras mutations and benefit from cetuximab in advanced colorectal cancer. N. Engl. J. Med. 359, 1757-1765. doi: 10.1056/NEJMoa0804385

Kelley, R. K., Wang, G., and Venook, A. P. (2011). Biomarker use in colorectal cancer therapy. J. Natl. Compr. Canc. Netw. 9, 1293-1302.

Liu, M., Wang, L., Bongartz, T., Hawse, J. R., Markovic, S. N., Schaid, D. J., et al. (2012). Aromatase inhibitors, estrogens and musculoskeletal pain: estrogendependent T-cell leukemia 1A (TCL1A) gene-mediated regulation of cytokine expression. Breast Cancer Res. 14, R41. doi: 10.1186/bcr3137

MacConaill, L. E., Van Hummelen, P., Meyerson, M., and Hahn, W. C. (2011). Clinical implementation of comprehensive strategies to characterize cancer genomes: opportunities and challenges. Cancer Discov. 1, 297-311. doi: 10.1158/2159-8290.CD-11-0110

Monte, A. A., Heard, K. J., and Vasiliou, V. (2012). Prediction of drug response and safety in clinical practice. J. Med. Toxicol. 8, 43-51. doi: 10.1007/s13181-0110198-7

Ng, K. P., Hillmer, A. M., Chuah, C. T., Juan, W. C., Ko, T. K., Teo, A. S., et al. (2012). A common BIM deletion polymorphism mediates intrinsic resistance and inferior responses to tyrosine kinase inhibitors in cancer. Nat. Med. 18, 521-528. doi: $10.1038 / \mathrm{nm} .2713$

Ong, F. S., Das, K., Wang, J., Vakil, H., Kuo, J. Z., Blackwell, W. L., et al. (2012). Personalized medicine and pharmacogenetic biomarkers: progress in molecular oncology testing. Expert Rev. Mol. Diagn. 12, 593-602. doi: 10.1586/erm.12.59

Onitilo, A. A., McCarty, C. A., Wilke, R. A., Glurich, I., Engel, J. M., Flockhart, D. A., et al. (2009). Estrogen receptor genotype is associated with risk of venous thromboembolism during tamoxifen therapy. Breast Cancer Res. Treat. 115, 643-650. doi: 10.1007/s10549-008-0264-2

Ou, S. H. (2011). Crizotinib: a novel and first-in-class multitargeted tyrosine kinase inhibitor for the treatment of anaplastic lymphoma kinase rearranged nonsmall cell lung cancer and beyond. Drug Des. Devel. Ther. 5, 471-485. doi: 10.2147/DDDT.S19045

Ou, S. H., Bartlett, C. H., Mino-Kenudson, M., Cui, J., and Iafrate, A. J. (2012). Crizotinib for the treatment of ALK-rearranged non-small cell lung cancer: a success story to usher in the second decade of molecular targeted therapy in oncology. Oncologist 17, 1351-1375. doi: 10.1634/theoncologist.2012-0311

Paugh, S. W., Stocco, G., McCorkle, J. R., Diouf, B., Crews, K. R., and Evans, W. E. (2011). Cancer pharmacogenomics. Clin. Pharmacol. Ther. 90, 461-466. doi: $10.1038 /$ clpt.2011.126
Ramsey, L. B., Bruun, G. H., Yang, W., Trevino, L. R., Vattathil, S., Scheet, P., et al. (2012). Rare versus common variants in pharmacogenetics: SLCO1B1 variation and methotrexate disposition. Genome Res. 22, 1-8. doi: 10.1101/gr.129668.111

Rehm, H. L., Bale, S. J., Bayrak-Toydemir, P., Berg, J. S., Brown, K. K., Deignan, J. L., et al. (2013). ACMG clinical laboratory standards for next-generation sequencing. Genet. Med. 15, 733-747. doi: 10.1038/gim.2013.92

Relling, M. V., Gardner, E. E., Sandborn, W. J., Schmiegelow, K., Pui, C. H., Yee, S. W., et al. (2011). Clinical Pharmacogenetics Implementation Consortium guidelines for thiopurine methyltransferase genotype and thiopurine dosing. Clin. Pharmacol. Ther. 89, 387-391. doi: 10.1038/clpt. 2010.320

Ritchie, M. D. (2012). The success of pharmacogenomics in moving genetic association studies from bench to bedside: study design and implementation of precision medicine in the post-GWAS era. Hum. Genet. 131, 1615-1626. doi: $10.1007 / \mathrm{s} 00439-012-1221-\mathrm{z}$

Simon, R. (2013). Drug-diagnostics co-development in oncology. Front. Oncol. 3:315. doi: $10.3389 /$ fonc. 2013.00315

Simon, R., and Polley, E. (2013). Clinical trials for precision oncology using nextgeneration sequencing. Pers. Med. 10, 485-495. doi: 10.2217/pme.13.36

Trevino, L. R., Shimasaki, N., Yang, W., Panetta, J. C., Cheng, C., Pei, D., et al. (2009). Germline genetic variation in an organic anion transporter polypeptide associated with methotrexate pharmacokinetics and clinical effects. J. Clin. Oncol. 27, 5972-5978. doi: 10.1200/JCO.2008.20.4156

Van Cutsem, E., Kohne, C. H., Hitre, E., Zaluski, J., Chang Chien, C. R., Makhson, A., et al. (2009). Cetuximab and chemotherapy as initial treatment for metastatic colorectal cancer. N. Engl. J. Med. 360, 1408-1417. doi: 10.1056/NEJMoa0805019

Wilke, R. A., Xu, H., Denny, J. C., Roden, D. M., Krauss, R. M., McCarty, C. A., et al. (2011). The emerging role of electronic medical records in pharmacogenomics. Clin. Pharmacol. Ther. 89, 379-386. doi: 10.1038/clpt.2010.260

Conflict of Interest Statement: The authors declare that the research was conducted in the absence of any commercial or financial relationships that could be construed as a potential conflict of interest.

Received: 04 March 2014; accepted: 21 March 2014; published online: 08 April 2014. Citation: Filipski KK, Mechanic LE, Long $R$ and Freedman AN (2014) Pharmacogenomics in oncology care. Front. Genet. 5:73. doi: 10.3389/fgene. 2014.00073

This article was submitted to Pharmacogenetics and Pharmacogenomics, a section of the journal Frontiers in Genetics.

Copyright (C) 2014 Filipski, Mechanic, Long and Freedman. This is an openaccess article distributed under the terms of the Creative Commons Attribution License (CC BY). The use, distribution or reproduction in other forums is permitted, provided the original author(s) or licensor are credited and that the original publication in this journal is cited, in accordance with accepted academic practice. No use, distribution or reproduction is permitted which does not comply with these terms. 